Supporting Information (part 2: figures)

\title{
Ultrafast polyphenol metabolomics of red wines using microLC-MS/MS
}

Yan $\mathrm{Ma}^{(1)}$, Nobuo Tanaka ${ }^{(1,2)}$, Arpana Vaniya ${ }^{(1)}$, Tobias Kind ${ }^{(1)}$ and Oliver Fiehn*(1,3)

(1) UC Davis Genome Center - Metabolomics, University of California, Davis, California 95616, United States

(2) GL Sciences Inc., Iruma, Saitama 358-0032,Japan

(3) Department of Biochemistry, Faculty of Sciences, King Abdulaziz University, Jeddah, Saudi-Arabia

*Corresponding author

Oliver Fiehn

Email address: ofiehn@ucdavis.edu

Phone: +1-530-754-8258

Fax: +1-530-754-9658

Figure S1. Orthogonal signal correction partial least squares (O-PLS) selection for the 10 most important features positively or negatively correlated to the average tasting score of an untrained panel of 14 adults. Selected features are colored in green. Pcorr was the correlation between LV1 scores and the original variables (red wine components).

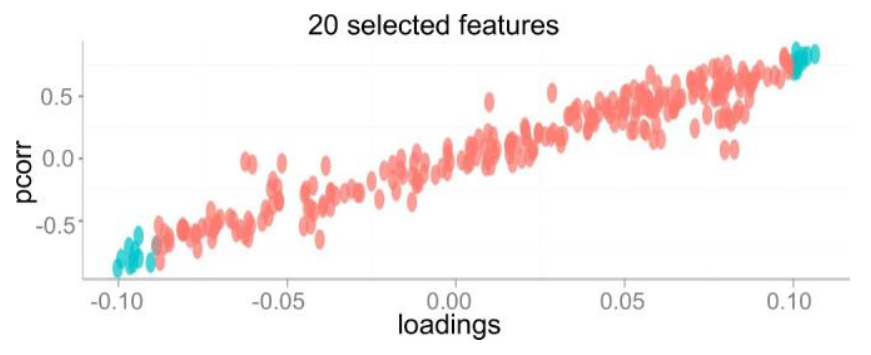

